\title{
A case of autonomous cortisol secretion in a patient with subclinical Cushing's syndrome, GNAS mutation, and paradoxical cortisol response to dexamethasone
}

Chihiro Sakaguchi ${ }^{1}$, Kenji Ashida ${ }^{1,2^{*}}$ (D) Kenichi Kohashi ${ }^{3}$, Kenji Ohe ${ }^{4}$, Yoichi Fujii ${ }^{5}$, Seiichi Yano ${ }^{1}$, Yayoi Matsuda', Shohei Sakamoto ${ }^{1}$, Ryuichi Sakamoto ${ }^{1}$, Yoshinao Oda ${ }^{3}$, Masatoshi Nomura ${ }^{1,2}$ and Yoshihiro Ogawa ${ }^{1}$

\begin{abstract}
Background: Increased urinary free cortisol in response to the oral administration of dexamethasone is a paradoxical reaction mainly reported in patients with primary pigmented nodular adrenocortical disease. Here, we describe the first case of subclinical Cushing's syndrome represented by autonomous cortisol secretion and paradoxical response to oral dexamethasone administration, harboring an activating mutation in the a subunit of the stimulatory G protein (GNAS).

Case presentation: A 65-year-old woman was diagnosed with subclinical Cushing's syndrome during an evaluation for bilateral adrenal masses. Tumors of unknown origin were found in the heart, brain, thyroid gland, colon, pancreas, and both adrenal glands. Adenocarcinoma of the sigmoid colon and systemic brown-patchy skin pigmentation were also present. Her urinary cortisol levels increased in response to oral dexamethasone, while serum dehydroepiandrosterone-sulfate was not suppressed. After right adrenalectomy, genetic analysis of the resected tumor revealed the somatic GNAS activating mutation, p.R201H. Paradoxical urinary cortisol response persisted even after unilateral adrenal resection, although serum and urinary cortisol levels were attenuated.

Conclusions: This patient harbored a GNAS activating mutation, and presented with a mild cortisol- and androgen-producing adrenal adenoma. Administration of oral dexamethasone paradoxically increased cortisol levels, possibly via the stimulation of the cyclic adenosine monophosphate-dependent protein kinase A signaling pathway, which is seen in patients with pigmented nodular adrenocortical disease or Carney complex. GNAS mutations may provide clues to the mechanisms of hyper-function and tumorigenesis in the adrenal cortex, especially in bilateral adrenal masses accompanied by multiple systemic tumors. Examining GNAS mutations could help physicians detect extra-adrenal malignancies, which may contribute to an improved prognosis for patients with this type of Cushing's syndrome.
\end{abstract}

Keywords: Cushing's syndrome, GNAS, Adrenal tumor, Cortisol, Cyclic-AMP, Protein kinase A

\footnotetext{
* Correspondence: ashida@med.kurume-u.ac.jp

${ }^{1}$ Department of Medicine and Bioregulatory Science, Graduate School of

Medical Sciences, Kyushu University, Fukuoka, Japan

${ }^{2}$ Division of Endocrinology and Metabolism, Department of Internal

Medicine, Kurume University School of Medicine, 67 Asahi-machi,

Kurume-city, Fukuoka 830-0011, Japan

Full list of author information is available at the end of the article
}

(c) The Author(s). 2019 Open Access This article is distributed under the terms of the Creative Commons Attribution 4.0 International License (http://creativecommons.org/licenses/by/4.0/), which permits unrestricted use, distribution, and reproduction in any medium, provided you give appropriate credit to the original author(s) and the source, provide a link to the Creative Commons license, and indicate if changes were made. The Creative Commons Public Domain Dedication waiver (http://creativecommons.org/publicdomain/zero/1.0/) applies to the data made available in this article, unless otherwise stated. 


\section{Background}

The cyclic adenosine monophosphate (cAMP)-dependent protein kinase A (PKA) signaling pathway is considered a mainstay for adrenal steroid production and cell proliferation. Several mutations in the cAMP-PKA pathway are responsible for autonomous production of glucocorticoids and adrenal tumorigenesis, resulting in Cushing's syndrome [1-5].

Inactivating mutation of the cAMP-dependent protein kinase type $1 \alpha$ regulatory subunit (PRKAR1A) is responsible for both primary pigmented nodular adrenocortical disease (PPNAD) and the Carney complex [6]. Paradoxical cortisol response to dexamethasone administration is a unique response observed in 69\% of PPNAD cases [7]. However, few reports have described this response $[8,9]$, and the underlying mechanisms remain unclear.

We describe a case of subclinical Cushing's syndrome (SCS) [10] with autonomous cortisol secreting adrenal tumor [11], who showed this paradoxical reaction due to harboring an activating mutation in the $\alpha$ subunit of the somatic stimulatory $\mathrm{G}$ protein (GNAS). Although GNAS mutations have been well-described in the McCune Albright syndrome [4], sporadic mutations of this gene have been reported in various neoplasms [12], including corticosteroid-producing adrenal tumors that can lead to Cushing's syndrome $[1-5,12]$. The present case may confirm and show features of autonomous steroid production and adrenocortical tumorigenesis leading to SCS via constitutive activation of the cAMP-dependent PKA pathway.

\section{Case presentation}

A 65-year-old woman was referred to our endocrinology center for evaluation of diabetes mellitus, hyperlipidemia, and bilateral adrenal masses, which were detected for the first time prior to a scheduled operation for sigmoid-colon cancer. She previously underwent total hysterectomy for uterine fibroids at the age of 44. She was on anti-hypertensive medication from approximately 40 years of age, and had experienced aortic dissection at the age of 56 . She showed normal stature and a body mass index of 24.7 $\mathrm{kg} / \mathrm{m}^{2}$. She did not show any Cushingoid signs. Fasting morning serum cortisol and urinary free cortisol levels (measured by immune radio metric assay method, SRL, Tokyo) were normal (Table 1). However, midnight levels of serum cortisol were high, and both overnight dexamethasone suppression tests, using $1 \mathrm{mg}$ and $8 \mathrm{mg}$ dexamethasone, did not suppress serum cortisol or dehydroepiandrosterone (DHEA) -sulfate levels. Plasma ACTH levels were low and did not respond to $100 \mu \mathrm{g}$ of intravenous corticotropin-releasing hormone. Furthermore, a dexamethasone suppression test using Liddle's method [7] showed a paradoxical increase in the levels of urinary cortisol (Table 2). The ratio of plasma aldosterone concentration (PAC) to plasma renin activity (PRA) was significantly high, although PAC was within the normal range. Based on the results of endocrinological examinations, the patient was diagnosed with idiopathic

Table 1 Laboratory data of the present patient

\begin{tabular}{|c|c|c|c|c|c|}
\hline Variable, unit & Value & Reference range & Variable, unit & Value & Reference range \\
\hline <Blood examination> & & & $<$ Urine analysis $>$ & & \\
\hline Fasting plasma glucose, mg/dL & 111 & 73-109 & Free cortisol, $\mu \mathrm{g} /$ day & 73.5 & $11.2-80.3$ \\
\hline Hemoglobin $A_{1} C, \%$ & 7.7 & $4.9-6.0$ & Androsterone, mg/day & 0.31 & $0.4-3.00$ \\
\hline $\mathrm{Na}, \mathrm{mmol} / \mathrm{L}$ & 143 & $138-145$ & Etiocholanolone, mg/day & 0.56 & $0.30-2.50$ \\
\hline $\mathrm{K}, \mathrm{mmol} / \mathrm{L}$ & 3.3 & $3.6-4.8$ & Dehydroepiandrosterone, mg/day & 0.02 & $0.04-2.60$ \\
\hline $\mathrm{Cl}, \mathrm{mmol} / \mathrm{L}$ & 105 & $101-108$ & 11-OH-Androsterone, mg/day & 0.4 & $0.22-1.60$ \\
\hline ACTH (0900 h), pg/mL & 3.1 & $7.2-63.3$ & 11-OH-Etiocholanorone, mg/day & 0.27 & $0.02-0.65$ \\
\hline ACTH (2300 h), pg/mL & 2.5 & N/A & 11-keto-Androsterone, mg/day & 0.01 & $<0.07$ \\
\hline Cortisol (0900 h), $\mu \mathrm{g} / \mathrm{dL}$ & 10.6 & $4.0-18.3$ & 11-keto-Etiocholanolone, mg/day & 0.1 & $0.03-0.50$ \\
\hline Cortisol (2300 h), $\mu \mathrm{g} / \mathrm{dL}$ & 11.4 & $<5.0$ & & & \\
\hline Dehydroepiandrosterone sulfate, $\mu \mathrm{g} / \mathrm{dL}$ & 129 & $12-133$ & $<$ Adrenal venous sampling $>$ & & \\
\hline Total testosterone, ng/mL & 0.26 & $0.11-0.47$ & Cortisol (rt. adrenal vein), $\mu \mathrm{g} / \mathrm{dL}$ & 361 & \\
\hline Corticosterone, $\mathrm{ng} / \mathrm{mL}$ & 2.36 & $0.12-8.48$ & Cortisol (It. adrenal vein), $\mu \mathrm{g} / \mathrm{dL}$ & 380 & \\
\hline Deoxycorticosterone, ng/mL & 0.09 & $0.03-0.33$ & Cortisol (inferior vena cava), $\mu \mathrm{g} / \mathrm{dL}$ & 33.6 & \\
\hline PRA, ng/mL/h & 0.2 & $0.3-2.9$ & PAC (rt. adrenal vein), $\mathrm{pg} / \mathrm{mL}$ & 10,900 & \\
\hline \multirow[t]{2}{*}{$\mathrm{PAC}, \mathrm{pg} / \mathrm{mL}$} & 88.3 & 29.9-159 & PAC (It. adrenal vein), pg/mL & 13,000 & \\
\hline & & & PAC (inferior vena cava), $\mathrm{pg} / \mathrm{mL}$ & 328 & \\
\hline
\end{tabular}

Adrenal venous sampling was performed while administering continuous intravenous infusion of ACTH (50 $\mu \mathrm{g} / \mathrm{hour})$. Text in parentheses indicate the location of blood sampling

ACTH, adrenocorticotropic hormone; PRA, plasma renin activity; PAC, plasma aldosterone concentration 
Table 2 Endocrinological data of loading test

\begin{tabular}{|c|c|c|c|c|}
\hline Loading agent & Variable, unit & $\begin{array}{l}\text { Value, pre- } \\
\text { operation }\end{array}$ & $\begin{array}{l}\text { Value, post- } \\
\text { operation }\end{array}$ & Reference range \\
\hline $1 \mathrm{mg}$ of DEX, overnight & Serum cortisol, $\mu \mathrm{g} / \mathrm{dL}$ & 11.8 & 4.6 & $<1.8$ \\
\hline $8 \mathrm{mg}$ of DEX, overnight & Serum cortisol, $\mu \mathrm{g} / \mathrm{dL}$ & 13.1 & 8.7 & $<1.0$ \\
\hline \multirow[t]{2}{*}{$100 \mu \mathrm{g}$ of CRH intravenously } & Basal ACTH, pg/mL & 2.7 & 2.6 & $7.2-63.3$ \\
\hline & $\begin{array}{l}\text { Peak ACTH, pg/mL (time, } \\
\text { min) }\end{array}$ & $5.2(90)$ & $9.1(90)$ & $\begin{array}{l}>2 \times \text { basal } \\
\text { ACTH }\end{array}$ \\
\hline \multirow[t]{2}{*}{$2 \mathrm{~L}$ of saline intravenously } & PAC (before loading), pg/mL & 78 & 73 & 29.9-159 \\
\hline & PAC (after loading), pg/mL & 92 & 81 & $<60$ \\
\hline \multirow[t]{6}{*}{50 mg of captopril orally } & $\mathrm{PAC}(0 \mathrm{~min}), \mathrm{pg} / \mathrm{mL}$ & 56 & 114 & $29.9-159$ \\
\hline & PAC (60 min), pg/mL & 59 & 102 & $\mathrm{~N} / \mathrm{A}$ \\
\hline & PAC (90 min), pg/mL & 50 & 82 & N/A \\
\hline & ARR (0 min) & 140 & 285 & $<200$ \\
\hline & ARR (60 min) & 590 & 102 & $<200$ \\
\hline & ARR (90 min) & 496 & 74 & $<200$ \\
\hline \multirow{3}{*}{$\begin{array}{l}40 \mathrm{mg} \text { of furosemide intravenously with keeping } \\
\text { upright }\end{array}$} & PRA (0 min), ng/mL/h & 0.3 & 0.4 & $0.3-2.9$ \\
\hline & PRA (60 min), ng/mL/h & 0.3 & 0.5 & $>2.0$ \\
\hline & PRA (120 min), ng/mL/h & 0.4 & 0.8 & $>2.0$ \\
\hline \multicolumn{5}{|l|}{$<$ Dexamethasone suppression test, Liddle's method> } \\
\hline No DEX & Urinary- free cortisol, $\mu \mathrm{g} /$ day & 74 & 14 & $11.2-80.3$ \\
\hline $2 \mathrm{mg} /$ day of DEX, the first day & Urinary- free cortisol, $\mu \mathrm{g} /$ day & 538 & 32 & \\
\hline $2 \mathrm{mg} /$ day of DEX, the second day & Urinary- free cortisol, $\mu \mathrm{g} /$ day & 284 & 28 & \\
\hline $8 \mathrm{mg} /$ day of DEX, the first day & Urinary- free cortisol, $\mu \mathrm{g} /$ day & 141 & 21 & \\
\hline $8 \mathrm{mg} /$ day of DEX, the second day & Urinary- free cortisol, $\mu \mathrm{g} /$ day & 136 & 20 & \\
\hline
\end{tabular}

Overnight suppression test with $1 \mathrm{mg}$ and $8 \mathrm{mg}$ of DEX did not suppress cortisol levels, but paradoxically resulted in an increase in urinary cortisol levels $\mathrm{DEX}$, dexamethasone; $\mathrm{CRH}$, corticotropin-releasing hormone; $\mathrm{ACTH}$, adrenocorticotropic hormone; PAC, plasma aldosterone concentration; ARR, plasma aldosterone / plasma renin activity ratio; PRA, plasma renin activity; N/A, not applicable

hyperaldosteronism [13] (Tables 1 and 2). Adrenal venous sampling indicated bilateral aldosterone hypersecretion (Table 1). Bilateral adrenal tumors, $25 \times 13 \mathrm{~mm}$ and $18 \times 15$ $\mathrm{mm}$, in the right and left gland respectively, had the appearance of adrenocortical adenoma on computed tomography (Fig. 1a, b) and magnetic resonance imaging (Fig. 1c-f). Accumulations of ${ }^{131}$ I-adosterol in adrenal tumors were observed on both sides, though predominantly on the left (Fig. $1 \mathrm{~g})$. Various extra-adrenal masses were detected in several imaging modalities, and patchy brown skin pigmentations were observed systemically (Fig. 2).

Thus, the patient was diagnosed with SCS [10] due to bilateral functioning autonomous cortisol secreting adrenal tumors [11]. Although serum cortisol and urinary free cortisol levels decreased after left unilateral laparoscopic adrenalectomy, the paradoxical response persisted (Table 2). Pathological examination revealed adrenocortical adenoma. The tumor consisted of round to polygonal-shaped cells with microvascular or eosinophilic cytoplasm, proliferating in an alveolar fashion, accompanied by hemorrhage, inflammatory infiltrate and lipochrome deposit, leading to the diagnosis of adrenal adenoma. Immunohistochemical analysis showed positive expression for cytochrome P450 (CYP) 17A1, HSD3B type-1, HSD3B type-2, dehydroepiandrosterone sulfotransferase, and CYP11B1, but not for CYP11B2 (Fig. 3). Genetic examination of the adrenal tumor revealed the somatic GNAS mutation p.R201H, which is known to be responsible for McCune-Albright syndrome, although sporadic GNAS mutations have also been reported [2]. No PRKAR1A mutation was detected in either the adrenal adenoma or the peripheral blood. The patient was treated with eplerenone, which had successfully ameliorated persistent hypertension and hypokalemia at her one year follow up visit.

\section{Genetic analysis}

Genomic DNA was extracted from fresh frozen adenoma tumor tissues and peripheral blood. Sureselect $\mathrm{Hu}$ man All Exon V6 (Agilent Technologies, Santa Clara, CA, USA) was used for exome capture followed by massive parallel sequencing on the Illumina platform (HiSeq2500; Illumina, San Diego, CA, USA). Sequence alignment and mutation calling were performed using 
(a)

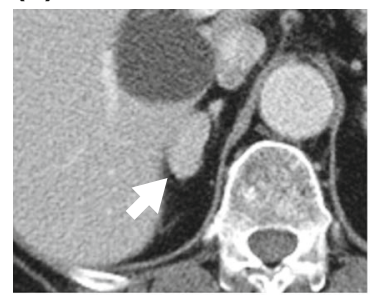

(c)

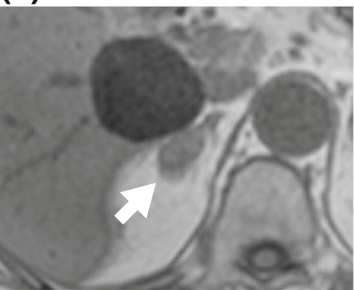

(e)

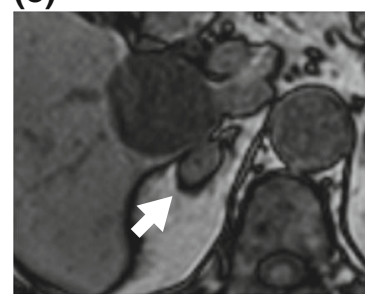

(b)

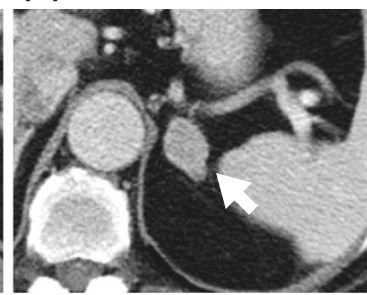

(d)

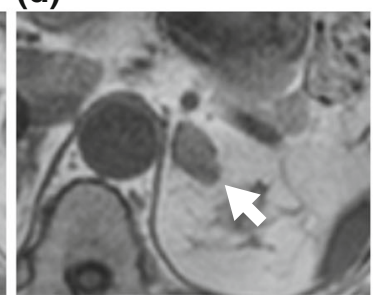

(f)

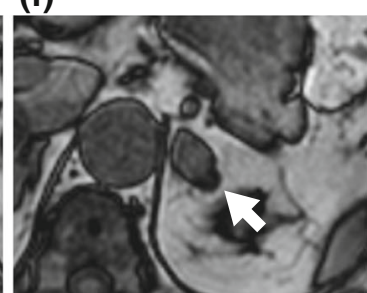

(g)

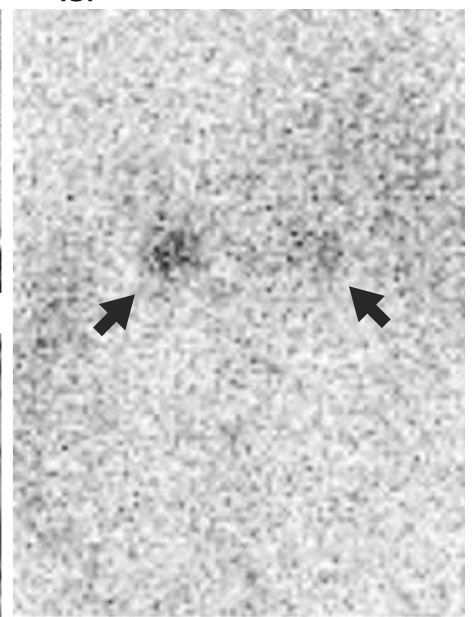

Fig. 1 Imaging study of bilateral adrenal masses. Computed tomography scan shows bilateral adrenal tumors. (a) right side, $25 \times 13 \mathrm{~mm}$ and (b) left side, $18 \times 15 \mathrm{~mm}$. Magnetic resonance imaging showed low intensity loci in adrenal tumors in "out phase" compared with "in phase" of chemical shift imaging on T1-weighted image. (c) right, in-phase; (d) right, out-phase; (e) left, in-phase; (f) left, out-phase. (g) ${ }^{131}{ }^{\text {I-adosterol }}$ scintigraphy shows bilateral adrenal accumulation. Arrows in each image designate the adrenal tumors or more accurately where the adosterol is accumulating in the adrenal tumors

our in-house pipeline, as previously described [2, 14] (Mean depth: 134.6 and 131.1). Candidate mutations for somatic mutations were filtered using the following criteria: (i) strand ratio $\neq 0,1$, (ii) number of variant reads in tumor sample $\geq 4$, (iii) number of variant reads in normal sample $\leq 1$, (iv) Fisher's exact $p<0.1$, (v) EBCall $p$ value $<10^{-4}$, (vi) variant allele frequency (VAF) in tumor sample $\geq 0.05$, (vii) annotated in exonic or splicing areas.

Candidate germline mutations with (i) strand ratio $\neq 0,1$, (ii) VAF between 0.4 and 0.6 , (iii) number of variant reads $\geq 4$, (iv) EBCall $\mathrm{p}$ value $<10^{-4}$ were further filtered by excluding synonymous variants and known variants with frequency of $\geq 0.1 \%$ in $1000 \mathrm{Ge}$ nomes Project (Nov. 2010 release), Exome Sequencing Project (ESP6500), and the Human Genome Variation Database (HGVD; October 2013 release).

Copy number analysis was performed using our in-house pipelines, CNACS [15], which could identify the copy number alterations (CNAs) using pooled normal samples as a reference.

As a result, we identified somatic GNAS p.R201H as the driver mutation of SCS (VAF: 0.379). No other somatic/germline mutations or CNAs was detected in any known causative genes including PRKAR1A.

\section{Discussion and conclusion}

This is the first reported case of SCS [10] due to autonomous cortisol producing adrenal adenoma [11] harboring a GNAS mutation, which exhibited the paradoxical cortisol response reported in patients with PPNAD. On immunohistochemical examinations, the resected adrenal adenoma showed potentials to produce cortisol and DHEA-sulfate, but not aldosterone. GNAS mutation-harboring Cushing's syndrome may provide clues to elucidate the mechanisms by which the glucocorticoid receptor (GR)-regulated steroidogenic enzymes operate in the adrenal gland, and the pathophysiology of SCS with extra-adrenal tumors.

Paradoxical cortisol response may provide a clue to predicting various mutations that enhance the cAMP-dependent PKA pathway, and to disclose the mechanisms which lead to the development of this type of Cushing's syndrome. Most cortisol-producing adrenocortical adenomas with paradoxical response reportedly harbor somatic PRKAR1A mutations [7, 16]. However, in a previous 
(a)

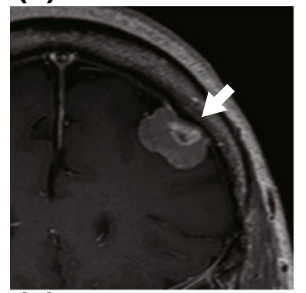

(c)

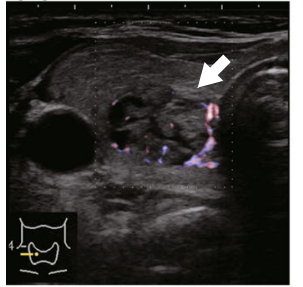

(e)

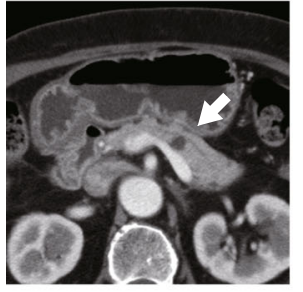

(b)

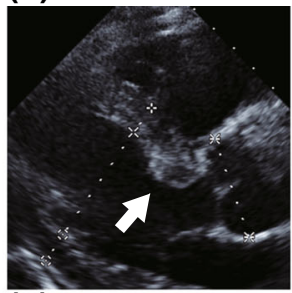

(d)

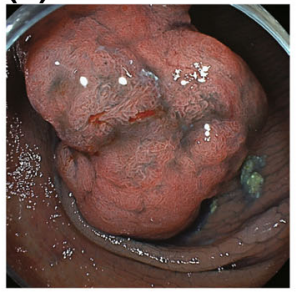

(f)

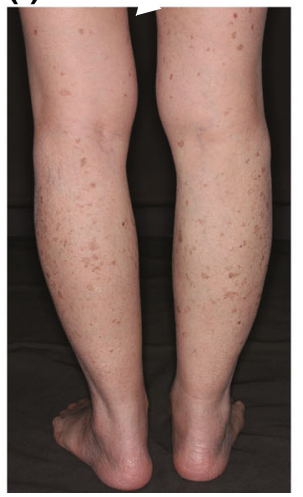

(g)

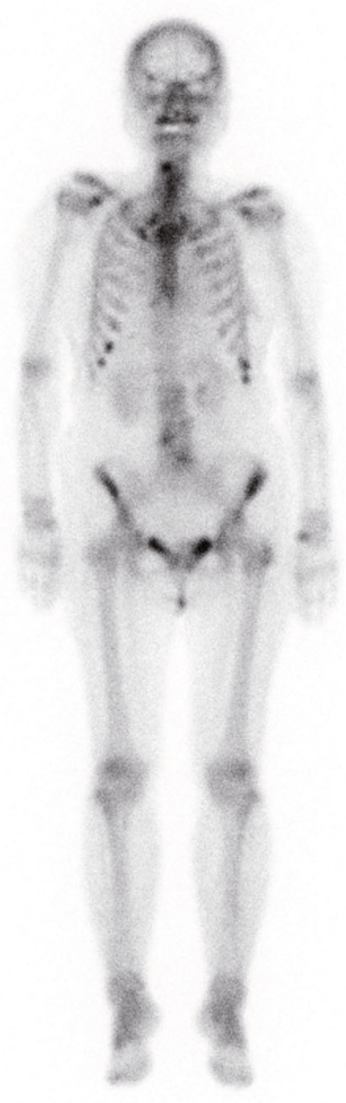

Fig. 2 Imaging studies of extra-adrenal tumors in this patient. a A convexity meningioma $21 \times 13 \times 18 \mathrm{~mm}$ in size on enhanced T1WI MRI of the Head (arrow). The pituitary tumor is not marked. b A hyperechoic lesion about $14 \mathrm{~mm}$ in size in the interventricular septum on echocardiography (arrow). c Cervical ultra-sonography revealed four tumors in both thyroid lobes (arrow). d Colonoscopy shows tubular adenocarcinoma in the colon. This tumor was later resected, but the status of GNAS mutation was not assessed. e Enhanced abdominal computed tomography showed cystic lesions compatible with intraductal papillary mucinous neoplasm in the pancreatic body (arrow). $\mathbf{f}$ Brown patchy pigmentations were observed systemically. Skin appearance with patchy pigmentations of bilateral lower limbs is shown. $\mathbf{g}$ X-ray imaging and bone scintigraphy with ${ }^{99 m}$ Tc-scintigraphy did not indicate fibrous dysplasia

study of patients with Cushing's syndrome, harboring somatic catalytic $\alpha$ subunit of PKA (PRKACA) or GNAS mutation, $17 / 20$ patients showed higher serum cortisol levels when treated with $8 \mathrm{mg}$ dexamethasone, but not with $1 \mathrm{mg}$ of dexamethasone (Table 2) [2], which is consistent with the present case. Thus, patients who exhibit high cortisol levels in response to high-dose dexamethasone might show paradoxical response. Furthermore, mildly elevated urinary free cortisol levels are detected when Liddle's test is performed in patients with ACTH-independent macronodular adrenal hyperplasia and/or adrenocortical cortisol-producing adenoma [17]. These patients present with aberrant expression and/or mutations of $\mathrm{G}$ protein-coupled receptors, both of which could enhance the cAMP-dependent PKA pathway [17]. In patients with PPNAD, the overexpression of GR in affected adrenal nodules [8] and the role of GR-stimulation in paradoxical response to dexamethasone [9] have been reported previously. GNAS mutations activate PKA via activating the cAMP-dependent signaling pathway, and subsequently enhance GR-dependent positive cortisol synthesis, which might lead to autonomous cortisol production [9].

The present case demonstrated the potential of the adrenal adenoma with GNAS mutation to produce cortisol and DHEA-sulfate, but not aldosterone. Immunohistochemical studies revealed that the adrenocortical adenoma in the present case expressed several steroidogenic enzymes including CYP 11B1, a cortisol synthetase (Fig. 3c-g), but not CYP11B2, an aldosterone synthetase (Fig. 3h). The presence of aldosterone-producing cell clusters suggested that the extra-tumor over-secretion was responsible for mild primary aldosteronism in the present case [18], although we did not look for GNAS mutation in the extra-tumor lesion in the adrenal cortex. Although the adrenal adenoma in this patient did not produce aldosterone, GNAS mutations have 
(a)

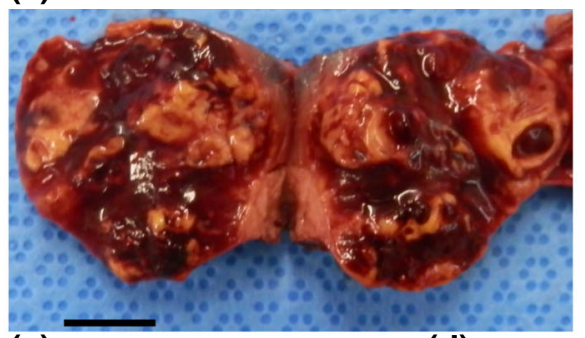

(c)

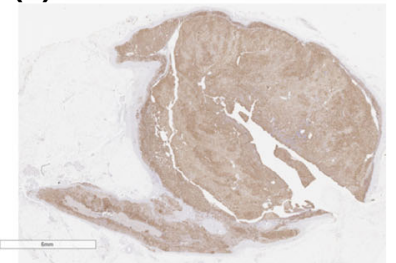

(f)

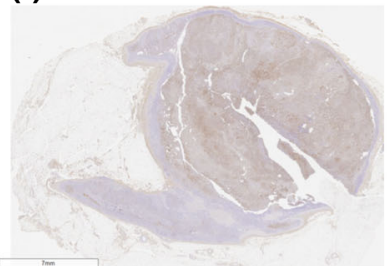

(d)

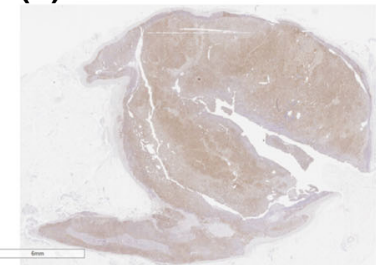

(g)

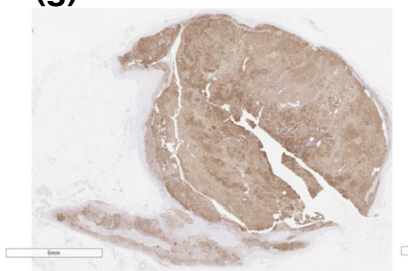

(b)

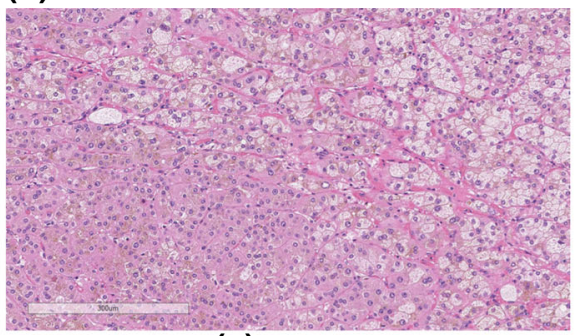

(e)

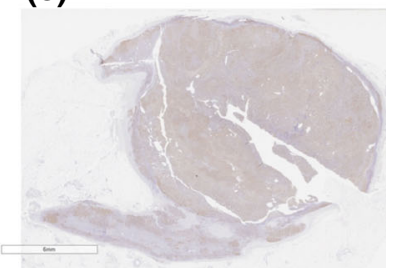

(h)

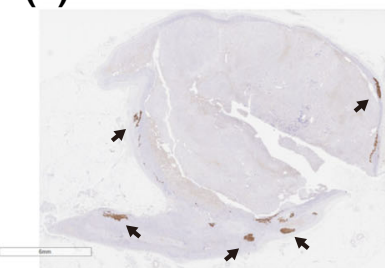

Fig. 3 Histopathological examination of adrenal adenoma. a Macro-image of the resected adrenal adenoma. b Micro-image with low magnitude using hematoxylin-eosin staining. The adrenal adenoma composed of compact cells and clear cells. $\mathbf{c}-\mathbf{h}$ Immunohistochemical staining was performed using anti-human antibodies as follows: c CYP17A1, d HSD3B type-1, e HSD3B type-2, f Dehydroepiandrosterone sulfotransferase, $\mathbf{g}$ CYP11B1, and $\mathbf{h}$ CYP11B2. The adrenal adenoma showed positive signal for cortisol producing enzymes and DHEA-sulfotransferase, which might be responsible for the high-normal serum level of DHEA-sulfate. CYP11B2 was absent in the adrenal tumors, but was positive in the extra-tumor area as aldosterone-producing cell cluster (arrows). Scale bars represent (a) $10 \mathrm{~mm},(\mathbf{b}) 300 \mu \mathrm{m},(\mathbf{c})-(\mathbf{e}) 6 \mathrm{~mm},(\mathbf{f}) 7 \mathrm{~mm}$, and (g) $6 \mathrm{~mm}$. CYP, cytochrome P450; HSD, hydroxysteroid dehydrogenase; CYP, cytochrome P450; HSD3B, 3ß-hydroxy- $\Delta 5$-steroid dehydrogenase.

been detected in adrenocortical adenomas producing both aldosterone and cortisol, the aldosterone-producing ability in these tumors has not been confirmed immunohistochemically [19] (Fig. 3h). However, the tumor was positive for DHEA-sulfotransferase (Fig. 3f), which could possibly explain the high-normal levels of DHEA-sulfate. Thus, it can be concluded that GNAS mutations may activate all steroidogenic enzymes except for those involved in aldosterone production. Our results suggest that cAMP-dependent PKA pathway is responsible for the production of androgen but not aldosterone in adrenal tumors.

Cushing's syndrome with paradoxical response to dexamethasone is known to present with a severe phenotype [9], because adrenocortical cells with cAMP-PKA signaling pathway mutation exhibit higher hormonal productivity [2]. Nonetheless, a mild form of Cushing's syndrome was reported in a case of ACTH-independent macronodular adrenal hyperplasia due to GNAS mutation [17]. Although we could not conclusively explain the reason for SCS in the present case, one plausible explanation could be the mosaic feature of mutated lesions [20], similar to the main genetic mechanism in McCune-Albright syndrome. Hence, further investigation of patients with McCune Albright syndrome, who had GNAS mutation-harboring Cushing's syndrome, could provide a better understanding of the pathophysiology of this type of Cushing's syndrome, which might even be considered as a partial form of McCune Albright syndrome [12, 21].

Various tumors were observed in the present case (Fig. 2). In a large case series study of patients with McCune-Albright syndrome [22], the prevalence of GNAS mutation-harboring Cushing's syndrome due to functional adrenal tumors was reported at $7.1 \%$. In another study a high prevalence of extra-adrenal malignancy was reported in patients with functioning bilateral adrenal tumors [23], with tumors being dispersed throughout the body, but the locations were similar to those in the present case, namely endometrium, breast, thyroid gland, and colon. Since we did not detect GNAS mutation in any other tissue, we could not conclude whether this case was a partial form of McCune Albright syndrome or a sporadic case of GNAS mutation identified in the adrenal adenoma [2, 12, 21]. On the other hand, enhanced signaling of the cAMP-PKA pathway 
due to GNAS and PRKAR1A mutations, leads to the activation of the $\mathrm{Wnt} / \beta$-catenin signaling pathway that might explain the pattern of endocrinological dysregulations and tumorigenesis in this case [12]. Our findings in the present case suggested that the activation of the cAMP-dependent PKA pathway in bilateral adrenocortical adenomas with paradoxical cortisol response and GNAS mutation, may result in multiple extra-adrenal neoplasms and malignancies [5, 24].

Additional cases would be required to support our results. In addition, there were a few limitations for genetic analysis in this study: WES and WES-based copy number analysis often miss structure variants and micro deletion/amplification. Due to this limitation, we might have missed germline mutations in some genes including PRKAR1A. Additional analysis with other platforms or sequencing other tumor tissues in this patient might help reach a comprehensive understanding of the molecular mechanisms in this kind of SCS with paradoxical cortisol response to oral dexamethasone and multifocal tumorigenesis.

In conclusion, this is the first reported case of SCS due to an autonomous cortisol secreting adrenal adenoma, harboring a GNAS mutation, which exhibited a paradoxical increase in urinary free cortisol levels in response to the oral administration of dexamethasone. This type of paradoxical response may provide a unique clue in the diagnosis of various types of cAMP-dependent PKA pathway-related Cushing's syndrome, including GNAS mutations, as the GNAS mutation-harboring adrenal tumor in this case showed potentials to produce cortisol and DHEA-sulfate, but not aldosterone. The possibility of GNAS mutations should be considered in patients with functional bilateral adrenal tumors to detect curable malignancies and contribute to better prognosis.

\section{Abbreviations \\ CAMP: Cyclic adenosine monophosphate; CNAs: Copy number alterations; CYP: Cytochrome P450; DHEA: Dehydroepiandrosterone; GNAS: Stimulatory G protein-a subunit; GR: Glucocorticoid receptor; PAC: Plasma aldosterone concentration; PKA: Protein kinase A; PPNAD: Pigmented nodular adrenocortical disease; PRA: Plasma renin activity; PRKACA: Catalytic alpha subunit of protein kinase A; PRKAR1A: Protein kinase type 1a regulatory subunit; SCS: Subclinical Cushing's syndrome; VAF: Variant allele frequency}

\section{Acknowledgments}

Prof. Hironobu Sasano (Tohoku University, Sendai, Japan) performed the immunohistochemical analysis and provided pathological suggestions to consider in our case discussion. We thank Editage (https://www.editage.jp/) for English editing.

\section{Funding}

The present study was supported by the Japan Agency for Medical Research and Development (AMED) under Grant Number JP17lk0201043 in the collection of data and in writing the manuscript.

\section{Availability of data and materials}

All data generated or analyzed during this study are included in this published article.

\section{Author's contributions}

CS and KA design the study and drafted the first manuscript. CS and KA contributed equally to the manuscript. CS collected the data. KK, and YF analyzed the adrenal tumor. $C S, K A, K K, K O, Y F$, and $M N$ interpreted the data. $K O, S Y, Y M, S S, R S, Y O 1, M N$, and $Y O 2$ provided input in the preparation of the manuscript. CS, KA, KO, YF, and MN revised the manuscript. All authors have read and approved the final version of manuscript.

\section{Ethics approval and consent to participate}

All procedures performed in this study were in accordance with the ethical standards of the Institutional Review Board of Kyushu university hospital and with the principles of the Declaration of Helsinki 2013. The patient provided informed consent, and approval by an ethics review board were exempted by the Institutional Review Board of Kyushu university hospital for this case report.

\section{Consent for publication}

Written informed consent for publication of the patient's clinical details and clinical images was obtained from the patient.

\section{Competing interests}

The authors declare that they have no competing interest.

\section{Publisher's Note}

Springer Nature remains neutral with regard to jurisdictional claims in published maps and institutional affiliations.

\section{Author details}

'Department of Medicine and Bioregulatory Science, Graduate School of Medical Sciences, Kyushu University, Fukuoka, Japan. ${ }^{2}$ Division of Endocrinology and Metabolism, Department of Internal Medicine, Kurume University School of Medicine, 67 Asahi-machi, Kurume-city, Fukuoka 830-0011, Japan. ${ }^{3}$ Department of Anatomic Pathology, Pathological Sciences, Graduate School of Medical Sciences, Kyushu University, Fukuoka, Japan. ${ }^{4}$ Faculty of Pharmaceutical Sciences, Fukuoka University, Fukuoka, Japan. ${ }^{5}$ Department of Pathology and Tumor Biology, Graduate School of Medicine, Kyoto University, Kyoto, Japan.

Received: 19 July 2018 Accepted: 16 January 2019

Published online: 22 January 2019

\section{References}

1. Goh G, Scholl UI, Healy JM, Choi M, Prasad ML, Nelson-Williams C, et al. Recurrent activating mutation in PRKACA in cortisol-producing adrenal tumors. Nat Genet. 2014;46:613-7. https://doi.org/10.1038/ng.2956.

2. Sato Y, Maekawa S, Ishii R, Sanada M, Morikawa T, Shiraishi Y, et al. Recurrent somatic mutations underlie corticotropin-independent Cushing's syndrome. Science. 2014;344:917-20. https://doi.org/10.1126/science.1252328.

3. Beuschlein F, Fassnacht M, Assié G, Calebiro D, Stratakis CA, Osswald A, et al. Constitutive activation of PKA catalytic subunit in adrenal Cushing's syndrome. N Engl J Med. 2014;370(11):1019-28. https://doi.org/10.1056/NEJMoa1310359.

4. Weinstein LS, Shenker A, Gejman PV, Merino MJ, Friedman E, Spiegel AM. Activating mutations of the stimulatory $\mathrm{G}$ protein in the McCune-Albright syndrome. N Engl J Med. 1991;325:1688-95. https://doi.org/10.1056/ NEJM199112123252403.

5. O'Hayre M, Vázquez-Prado J, Kufareva I, Stawiski EW, Handel TM, Seshagiri $S$, et al. The emerging mutational landscape of $\mathrm{G}$ proteins and G-protein-coupled receptors in cancer. Nat Rev Cancer. 2013;13: 412-24. https://doi.org/10.1038/nrc3521.

6. Stratakis CA, Kirschner LS. Clinical and genetic analysis of primary bilateral adrenal diseases (micro- and macronodular disease) leading to Cushing syndrome. Horm Metab Res. 1998;30:456-63.

7. Stratakis CA, Sarlis N, Kirschner LS, Carney JA, Doppman JL, Nieman LK, et al. Paradoxical response to dexamethasone in the diagnosis of primary pigmented nodular adrenocortical disease. Ann Intern Med. 1999;131:58591. https://doi.org/10.7326/0003-4819-131-8-199910190-00041.

8. Bourdeau I, Lacroix A, Schürch W, Caron P, Antakly T, Stratakis CA. Primary pigmented nodular adrenocortical disease: paradoxical responses of cortisol secretion to dexamethasone occur in vitro and are associated with increased expression of the glucocorticoid receptor. J Clin Endocrinol Metab. 2003;88:3931-7. https://doi.org/10.1210/jc.2002-022001. 
9. Louiset E, Stratakis CA, Perraudin V, Griffin K, Libé R, Cabrol S, et al. The paradoxical increase in cortisol secretion induced by dexamethasone in primary pigmented nodular adrenocortical disease involves a glucocorticoid receptormediated effect of dexamethasone on protein kinase a catalytic subunits. J Clin Endocrinol Metab. 2009;94:2406-13. https://doi.org/10.1210/jc.2009-0031.

10. Yanase T, Oki Y, Katabami T, Otsuki M, Kageyama K, Tanaka T, et al. New diagnostic criteria of adrenal subclinical Cushing's syndrome: opinion from the Japan Endocrine Society. Endocr J. 2018;65:383-93. https://doi.org/10. 1507/endocrj.EJ17-0456.

11. Fassnacht M, Arlt W, Bancos I, Dralle H, Newell-Price J, Sahdev A, et al. Management of adrenal incidentalomas: European Society of Endocrinology Clinical Practice Guideline in collaboration with the European network for the study of adrenal tumors. Eur J Endocrinol. 2016;175:G1-G34. https://doi.org/10.1530/EJE-16-0467.

12. Salpea P. Stratakis CA. Carney complex and McCune Albright syndrome: an overview of clinical manifestations and human molecular genetics. Mol Cell Endocrinol. 2014;386:85-91. https://doi.org/10.1016/.mce.2013.08.022.

13. Funder JW, Carey RM, Mantero F, Murad MH, Reincke M, Shibata H, et al. The Management of Primary Aldosteronism: case detection, diagnosis, and treatment: an Endocrine Society clinical practice guideline. J Clin Endocrinol Metab. 2016;101:1889-916. https://doi.org/10.1210/jc.2015-4061.

14. Shiraishi $Y$, Sato Y, Chiba K, Okuno Y, Nagata Y, Yoshida K, et al. An empirical Bayesian framework for somatic mutation detection from cancer genome sequencing data. Nucleic Acids Res. 2013:41:e89. https://doi.org/10.1093/nar/gkt126.

15. Yoshizato T, Nannya Y, Atsuta Y, Shiozawa Y, lijima-Yamashita Y, Yoshida K, et al. Genetic abnormalities in myelodysplasia and secondary acute myeloid leukemia: impact on outcome of stem cell transplantation. Blood. 2017;129: 2347-58. https://doi.org/10.1182/blood-2016-12-754796.

16. Bertherat J, Groussin L, Sandrini F, Matyakhina L, Bei T, Stergiopoulos S, et al. Molecular and functional analysis of PRKAR1A and its locus (17q22-24) in sporadic adrenocortical tumors: $17 \mathrm{q}$ losses, somatic mutations, and protein kinase a expression and activity. Cancer Res. 2003:63:5308-19.

17. Hsiao HP, Kirschner LS, Bourdeau I, Keil MF, Boikos SA, Verma S, et al. Clinical and genetic heterogeneity, overlap with other tumor syndromes, and atypical glucocorticoid hormone secretion in adrenocorticotropin-independent macronodular adrenal hyperplasia compared with other adrenocortical tumors. J Clin Endocrinol Metab. 2009:94:2930-7. https://doi.org/10.1210/jc.2009-0516.

18. Tokumoto M, Onoda N, Tauchi Y, Kashiwagi S, Noda S, Toi N, et al. A case of Adrenocoricotrophic hormone -independent bilateral adrenocortical macronodular hyperplasia concomitant with primary aldosteronism. BMC Surg. 2017;17:97. https://doi.org/10.1186/s12893-017-0293-z.

19. Nakajima Y, Okamura T, Horiguchi K, Gohko T, Miyamoto T, Satoh T, et al. GNAS mutations in adrenal aldosterone-producing adenomas. Endocr J. 2016;63:199-204. https://doi.org/10.1507/endocri.EJ15-0642.

20. Dumitrescu CE, Collins MT. McCune-Albright syndrome. Orphanet J Rare Dis. 2008;3:12

21. Lumbroso S, Paris F, Sultan C. Activating Gsalpha mutations: analysis of 113 patients with signs of McCune-Albright syndrome-a European collaborative study. J Clin Endocrinol Metab. 2004;89:2107-13. https:/doi.org/10.1210/jc.2003-031225.

22. Brown RJ, Kelly MH, Collins MT. Cushing syndrome in the McCuneAlbright syndrome. J Clin Endocrinol Metab. 2010;95:1508-15. https:// doi.org/10.1210/jc2009-2321.

23. Kawate H, Kohno M, Matsuda Y, Akehi Y, Tanabe M, Horiuchi T, et al. Longterm study of subclinical Cushing's syndrome shows high prevalence of extraadrenal malignancy in patients with functioning bilateral adrenal tumors. Endocr J. 2014;61:1205-12. https://doi.org/10.1507/endocrj.EJ14-0155.

24. Forbes SA, Bindal N, Bamford S, Cole C, Kok CY, Beare D, et al. COSMIC: mining complete cancer genomes in the catalogue of somatic mutations in Cancer. Nucleic Acids Res. 2011:39(Database issue):D945-50.

\section{Ready to submit your research? Choose BMC and benefit from:}

- fast, convenient online submission

- thorough peer review by experienced researchers in your field

- rapid publication on acceptance

- support for research data, including large and complex data types

- gold Open Access which fosters wider collaboration and increased citations

- maximum visibility for your research: over $100 \mathrm{M}$ website views per year

At $\mathrm{BMC}$, research is always in progress.

Learn more biomedcentral.com/submissions 